\title{
Pig Breeds Classification using Neuro-Statistic Model
}

\author{
Satyendra Nath Mandal ${ }^{1}$, Sanket Dan ${ }^{1}$, Pritam Ghosh ${ }^{1}$, Subhranil Mustafi ${ }^{1}$, Kunal Roy $^{1}$, \\ Kaushik Mukherjee ${ }^{1}$, Dilip Kumar Hajra ${ }^{2}$ and Santanu Banik ${ }^{3}$ \\ ${ }^{1}$ Kalyani Government Engineering College, Kalyani, Nadia (W.B)-741235 \\ ${ }^{2}$ Department of Agronomy, Faculty of Agriculture, UBKV, Pundibari, Cooch Behar, WB-736165 \\ ${ }^{3}$ ICAR-National Research Centre on Pig, Rani, Guwahati, Assam-781131 \\ E-mail: ${ }^{1}$ satyen_kgec@rediffmail.com
}

\begin{abstract}
Image classification using fully connected neural network is not efficient due to huge number of parameters in each layer. In this paper, we propose a Neuro-Statistic model for classification of five different pig breeds from pig images. The model consists of four sub modules which work together as a layered structure. We captured multiple individual pig images of five different pig breeds from different organized farms to conduct this research, segmented the captured pig images using hue based segmentation algorithm and then calculated the statistical properties like entropy, standard deviation, variance, mean, median, mode and color properties like H.S.V from the content of the individual segmented images. We fed all the extracted properties into Neural Network for Pig Breed (NNPB) to perform pig breed prediction with the classification module and analyzed the best performance, regression error plot, Error histogram and training state of NNPB. The performance of NNPB network was accepted based on error analysis and finally, we used the trained model to predict the breed of 50 pig images and achieved the prediction accuracy of $90 \%$.
\end{abstract}

Keywords: Classification, Regression, Histogram, Segmentation, Entropy

\section{INTRODUCTION}

The animal breeds' classification is still a challenging task and there is no unique method that provides a robust and efficient solution to all situations (Islam, M.P., \& Morimoto, T., 2017). The aim of classification is to assign a query object to one of class from a set of predefined classes based on the attribute values of the object. Artificial neural network (ANN) is widely used for classification of categorical data sets. The ANN uses many neurons which are computational units, connected with weighted links, inputs and biases. The neurons are producing output through activation functions and are transmitting it to the neurons at the next layer. The pattern which is to be classified is being fed into the network as input. The pattern will be processed through the network and produce some output. The error which will be calculated between predefined output and produced output. The training is to be performed to reduce error by adjusting weights of links associated layers and pattern classification neural network will be developed.
The developed model will be validated by the predefined input patters and their assigned classes for tuning the hyperparameters. Finally, the model will be applied on test dataset for finalization. But, image classification using artificial neural network is computationally poor compared to data oriented classifications. The image is stored in computer as 2D matrix of pixels and each pixel is fed into input layer for image classification. The number of inputs is equal to the product of rows and column of 2D matrix. The same numbers of weights are also associated for input connections from input to a single neuron in the hidden layer. Generally, the fully connected neural network is used for classification. All hidden layers have same number of neurons and the number of neurons in the problem space is dependent on number of input parameters. The number of weights in fully connected layer with 1000 neurons for image is something like $150 \mathrm{M}$ for one layer. That is why the image classification using fully connected neural network is not efficient. Most of the researcher used convolutional neural network (CNN) for image classifications. The researchers 
have used pre-trained model for classifying their images in transfer learning fashion where the features extraction part remains unchanged. They do not modify the feature extractions part and features of existing models to classify their own images. This problem motivated us to find new ways to classify the images based on the features extracted from its content. The aim of this paper is to develop pig breeds classification system based on statistical properties of the content of breed images and their color components. The five pig breeds named Yorkshire, Ghungroo, Hampshire, Mali and Duroc were captured from three organized pig farms. An arrangement was created at the time of capturing pigs to make the uniform background for all the pig images. The pigs kept moving freely and their site profile were captured by mobile phones with $10 \mathrm{MP}$ cameras. The 50 pigs were captured from each pig breed. We have segmented the pig images using hue based segmentation algorithms by us. The captured images are stored in computer memory as 2D matrix of pixels. We have extracted the statistical properties of the content of images like mean, median, mode, standard deviation, variance and color components like H, S and V values, from each segmented breed images. All computed statistical properties are fed into neural network and trained using supervised learning for mapping statistical properties with breed classes. We have developed a neural network for pig breed classification named NNPB which takes statistical parameters and color components as inputs and produces a single numeric value as output which is then categorized into pig breeds using the classification module. Out of 50 images for each breed, we have used 30 images for training the network, 10 for validating it and finally, 10 images for testing the network. We have used Matlab 2016 for extracting the values of the statistical parameters, color components and neural network. Finally, we used the trained model to predict the breed of 50 pig images and achieved the prediction accuracy of $90 \%$. This type of work has not been done before in pig breeds classification. This is the reason for making this paper. The rest of the paper is organized into 7 sections excluding Section 1 which is Introduction. Section 2 is about the related works relevant to this paper. Section 3 describes the theoretical background needed for this paper. Section 4 \& 5 explains the methods, tools and dataset used in this paper. Section 6 showcases the details of the results obtained and its implication. Section 7 consists of the concluding remarks and lastly the acknowledgements are given in Section 8 followed by the references.

\section{RELATED WORK}

Extraction and analysis of statistical features from images have been studied for a long time. Measuring statistical properties of pixel pairs at several distances in the image has been used for texture analysis (Wu \& Chen, 1992). Image classification based on first and second order statistics calculated from digital images (Kahou \& Sulema, 2010) and using such features to train neural image classifiers (Garg, Malhotra, \& Singh, 2018) have been attempted. It is very important to measure the effect of such statistical measures on digital image processing (Kumar \& Gupta, 2012) because it can be used to classify normal and abnormal mammograms (Youssef, Rabeh, Zbitou, \& Belaguid, 2014). Specific applications of using statistical parameters as features include content based image retrieval (Thilagam \& Arunesh, 2019) characterize images based on brightness distribution (Al-Ani \& Alheeti, 2017) which describes the lighting condition in any image.

Various statistical parameters have been used to differentiate between human finger knuckles and acts as a high precision biometrics for individual human identification (Akheela \& Naz, 2015).

Surface texture analysis using statistical-texture-features like surface morphology has been used to differentiate between defective and non-defective drug tablets based on external factors like temperature, humidity and moisture (Tahir \& Fahiem, 2014). Thus it is seen that external factors can affect the statistical features of an image. With the large scale acceptance artificial neural networks as efficient feature extractors, it has been applied to fields like occupational stress analysis and prediction (Pandey, Saxena, \& Bhatt, 2015) and prediction of inside air temperature of pillar coolers based on parameters like outside temperature, watering and airing (Islam \& Morimoto, 2017). Also animal classification systems based purely on convolutional neural networks has also been established (Trnovszký, Kamencay, Orješek, Benčo, \& Sýkora, 2017). The comparison of image classification techniques based on CNNs and SVMs establishes that CNNs are better than SVMs with respect to multiclass image classifications (Jawale, 2019). 


\section{ARTIFICIAL NEURAL NETWORK, STATISTICAL PARAMETERS AND IMAGE CLASSIFICATION}

\section{Artificial NeURAl Network}

An artificial neural network (ANN) is an efficient information processing system which resembles in characteristics with a biological neural network. ANN's possess large number of highly interconnected processing elements called neurons. Each neuron is connected with each other by a connection link. Each connection link is associated with a weight which contains information about the input signal. This information is used by the neural net to solve a particular problem. The neuron computes the weighted sum of all inputs along with their connecting weights and biases. The result is fed into activation function which is fed into next neurons of the next layer. Finally, the output is produced from output layers. The supervised learning is used in classification problems. The error is calculated between target and actual output produced by network based on inputs and weights. The error is reduced by updating the weights of connections among the layers known as training the network. The training is an iterative process and the iteration is stopped either when the maximum value is reached or the error reaches a steady state for successive iterations. The basic structure of a neural network model is depicted in Figure 1.

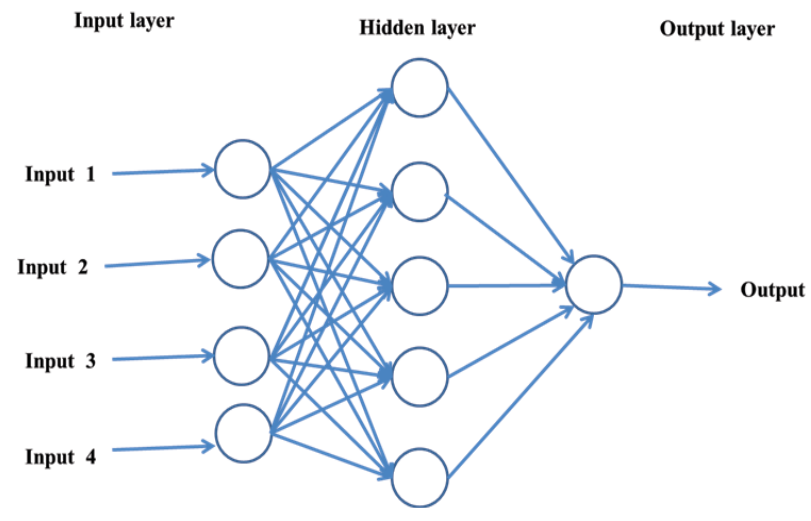

Fig. 1: Artificial Neural Network

\section{Training Algorithm of Artificial Neural Network}

In every iteration the weights of the connections associated with inputs and layers are updated until stopping conditions are reached. The connection weights are updated by the guidance of training functions. In this paper, LevenbergMarquardt algorithm (trainlm) training algorithm is used to update weights of connections. The Levenberg-Marquardt algorithm uses the approximation to the Hessian matrix. The Hessian matrix can be approximated as:

$\mathrm{H}=\mathrm{J}^{\mathrm{T}} \mathrm{J}$

And the gradient can be computed as:

$\mathrm{g}=\mathrm{J}^{\mathrm{T}} \mathrm{e}$

Where J is the Jacobian matrix that contains first derivatives of the network errors with respect to the weights and biases, and $e$ is a vector of network errors.

The Levenberg-Marquardt algorithm is defined as:

$$
\mathrm{x}_{\mathrm{k}+1}=\mathrm{x}_{\mathrm{k}}-\left[\mathrm{J}^{\mathrm{T}} \mathrm{J}+\mu \mathrm{I}\right]^{-1} \mathrm{~J}^{\mathrm{T}} \mathrm{e}
$$

When the scalar $\mu$ is zero, this is just Newton's method, using the approximate Hessian matrix. When $\mu$ is large, this becomes gradient descent with a small step size.

\section{Statistical Parameters and HSV Color COMPONENTS}

The various statistical measurements on content and color component of images are computed for classification. The gray scale images are stored as $2 \mathrm{D}$ matrix in memory. The details of parameters are furnished in this paper.

\section{H,S and V Components of HSV Color Model}

In RGB color model, red, green and blue lights are mixed together in various ratios to reproduce a broad array of colors (Program to Change RGB color model to HSV color model). The artists are used HSV (hue, saturation, value) color model for thinking about a color in terms of hue and saturation and values. The transformation from RGB to HSV as follows: 


\section{Pig Breeds Classification using Neuro-Statistic Model}

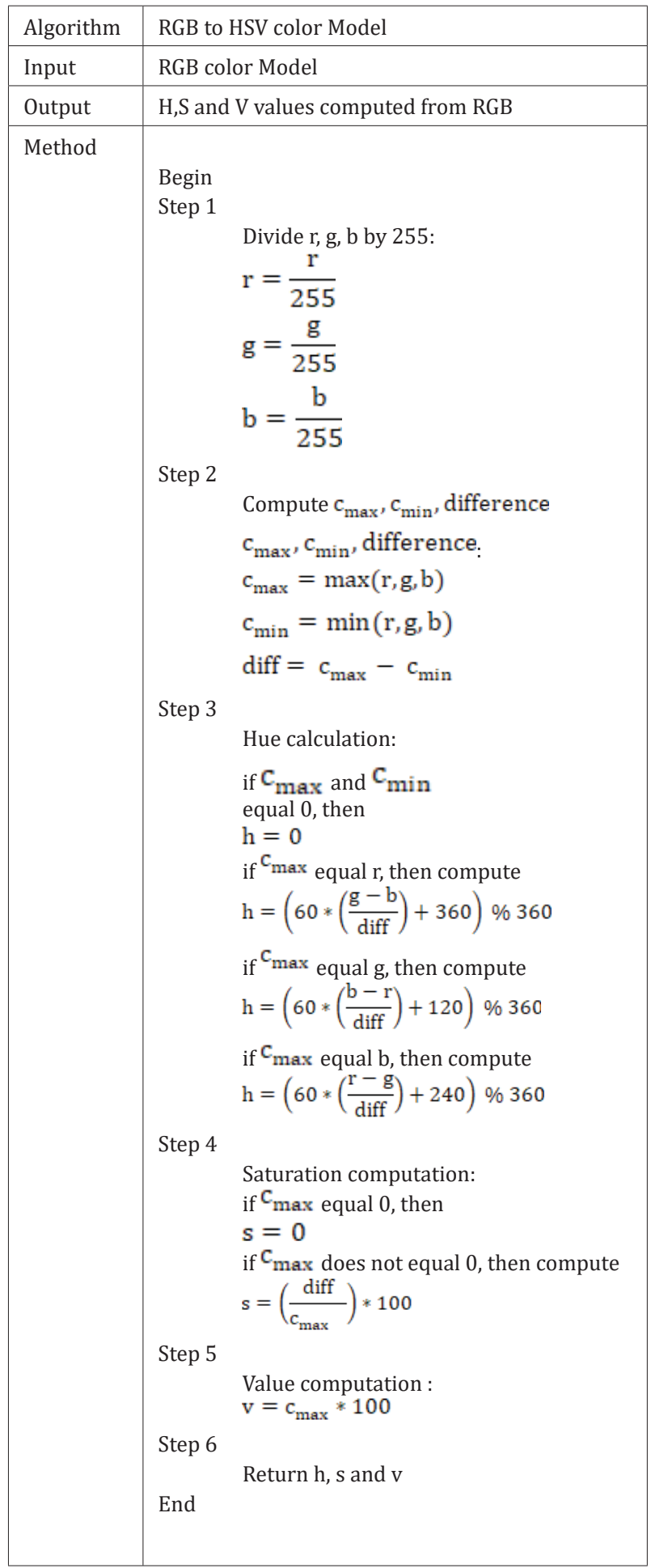

\section{Statistical Parameters}

\section{Entropy of Image}

Entropy is the statistical measurement of randomness and it may be used for characterization the texture of the input image. The entropy of an image is defined as follows:

$$
-\sum_{i=0}^{n-1} p_{i} \log _{b} p_{i}
$$

where $\mathrm{n}$ is the number of gray levels (256 for 8-bit images), $p_{i}$ is the probability of a pixel having gray level $i$, and $b$ is the base of the logarithm function.

\section{Standard Deviation}

The unbiased estimate of the standard deviation, $\mathrm{s}_{\mathrm{a}}$ of the brightness's within a region $(\Re)$ with $\Lambda$ pixels is called the sample standard deviation (Image Processing Fundamentals) and is given by:

$$
\begin{aligned}
& \mathrm{S}_{\mathrm{a}}=\sqrt{\frac{1}{\Lambda-1} \sum_{\mathrm{m}, \mathrm{n} \in \mathfrak{\Re}}\left(\mathrm{a}[\mathrm{m}, \mathrm{n}]-\mathrm{m}_{\mathrm{a}}\right)^{2}} \\
& =\sqrt{\frac{\sum_{\mathrm{m}, \mathrm{n} \in \mathrm{r}} \mathrm{a}^{2}[\mathrm{~m}, \mathrm{n}]-\Lambda \mathrm{m}_{\mathrm{a}}^{2}}{\Lambda-1}}
\end{aligned}
$$

\section{Mean, Sum, Max and Min of Image}

The mean intensity or brightness of an image is defined as the sample mean of the pixel intensities or brightness of image (Garg, M., Malhotra, M., \& Singh, H. 2018) ). The mean, $\mathrm{m}_{\mathrm{a}}$ of the intensities over the $\Lambda$ pixels of Image is given by:

$$
\mathrm{m}_{\mathrm{a}}=\frac{1}{\Lambda} \sum_{\mathrm{m}, \mathrm{n} \in \mathrm{r}} \mathrm{a}[\mathrm{m}, \mathrm{n}]
$$

The min, max and sum of intensities of image are defined by:

$$
\begin{aligned}
& \tilde{\mathrm{f}}(\mathrm{x}, \mathrm{y})=\min \{\mathrm{g}(\mathrm{r}, \mathrm{c}) \mid(\mathrm{r}, \mathrm{c}) \in \mathrm{W}\} \\
& \tilde{\mathrm{f}}(\mathrm{x}, \mathrm{y})=\max \{\mathrm{g}(\mathrm{r}, \mathrm{c}) \mid(\mathrm{r}, \mathrm{c}) \in \mathrm{W}\} \\
& \tilde{\mathrm{F}}(\mathrm{x}, \mathrm{y})=\operatorname{sum}\{\mathrm{g}(\mathrm{r}, \mathrm{c}) \mid(\mathrm{r}, \mathrm{c}) \in \mathrm{W}\}
\end{aligned}
$$

Where $\mathrm{g}$ is the gray value or intensity of pixel at location ( $\mathrm{x}$, $\mathrm{y}), \mathrm{r}$ and $\mathrm{c}$ are row and column of image size $\mathrm{W}$. 


\section{Variance of Image}

The general equation for calculating the mean, $\mu$, of a set of numbers, $\mathrm{X} 1$ - XN, would be written like this:

$$
\mu=\frac{\sum_{i=1}^{N} X_{i}}{N}=\frac{\sum X}{N}
$$

The variance $\left(\sigma^{2}\right)$ is defined as the sum of the squared distances of each term in the distribution from the mean $(\mu)$, divided by the number of terms in the distribution $(\mathrm{N})$ (Variance \& Standard Deviation).

\section{Median and Mode}

If the pixels are arranged in order either in ascending or descending of their intensities values, the middle value is call median. The image is containing $\mathrm{n}$ pixels, the median is written as:

$$
\begin{aligned}
& \text { Median }=\left(\frac{n+1}{2}\right)^{\text {th }} \text { pixel, if } n \text { is odd } \\
& \text { Or } \\
& \text { Median }=\left(\frac{n}{2}\right)^{\text {th }} \text { pixel, if } n \text { is even }
\end{aligned}
$$

The mode is the intensity value of pixels of an image which occurs with largest frequencies.

\section{PROPOSED MODEL AND MODULES}

\section{Proposed Model for Pig BReEd RECOGNITION}

In this paper, we have proposed the pig breed classification model comprising of a number of sub models in layered structure. Pigs from five different pig breeds were captured from organized pig farms using some restrictions. We have segmented the individual pig images and extracted statistical parameter values and H, S, V color components from the segmented pig images. We have developed the neural network for pig breed named NNPB for fitting the values of statistical parameters and color components with the pig image from five classes. The classification model is used to categorize the output of NNBP module and assigned the class among five classes of Pig breeds. Finally, the breed of pig is declared based on values of statistical parameters and color components of the input pig image as shown in Figure 2.

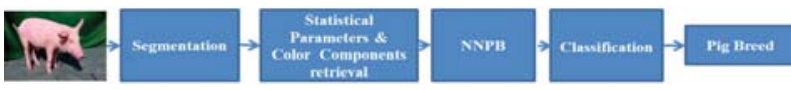

Fig. 2: Proposed Model for Pig Breed Classification

\section{Pig Image Segmentation}

We converted the captured images into equal size. Every pixel of an image was converted to HSV color model. The Hue was determined by keeping the Saturation and Value constant for each breed of pig taken in the study by excluding background of a photo. The HSV image was then converted to binary image with the help of calculated Hue. In the binary image, the visible parts of the pig in original picture turned to white while the background turned to black. The black and white binary image thus obtained might contain some white blobs. The areas of white blobs were measured. Except largest blob, all others blobs were inverted to black to grow a mask image. The obtained mask image was overlapped with original RGB image to get the segmented image of the pig as shown in Figure 3.

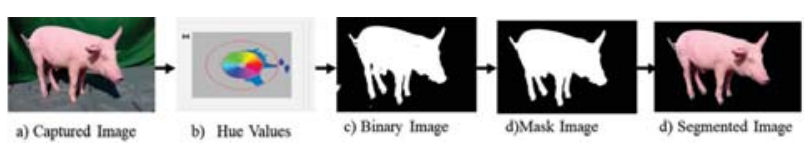

Fig. 3: Hue Calculation and Segmentation of Pig

\section{NeURAL NeTWORK FOR Pig BREEDS Classification}

To classify breed from pig images, feed forward neural network (known as 'fitnet') is used to fit an input-output relationship. Ten (10) parameter values like hue, saturation, value, entropy, standard deviation, mean, median, mode, sum and variance are calculated from the content of each segmented image from five different breeds. The ten different parameter values are then fed into the network's input layer. One hidden layer with ten neurons and the output layer with one neuron are used in this network. The tangent sigmoid transfer function (tansig) is used at hidden layer and linear transfer function is used at output layer. The LevenbergMarquardt algorithm (trainlm) training algorithm is used to update weights of connections and maximum iteration is set as 1000 as shown in Figure 4. 


\section{Pig Breeds Classification using Neuro-Statistic Model}

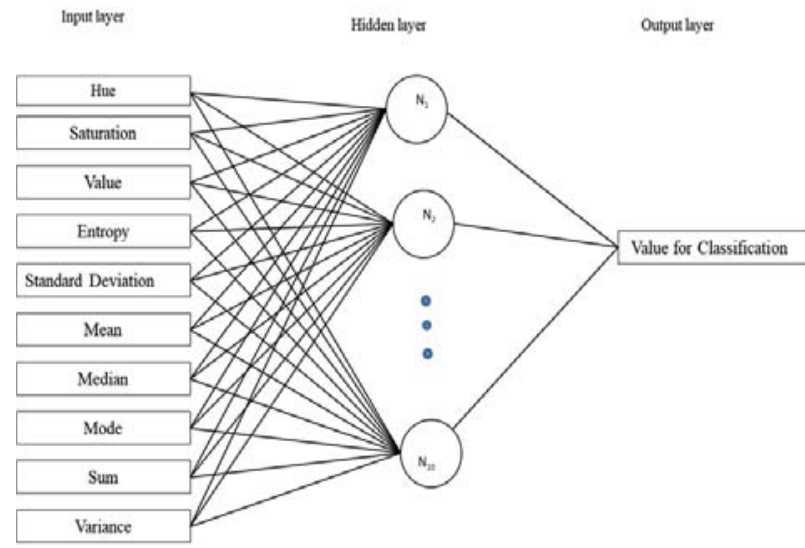

Fig. 4: Neural Network for Pig Breed Classification

\section{Classification module}

The supervised neural network is used in this paper. The pig breeds and their class assignments are given in Table 1.

Table 1: Pig Breed and Assigned Class

\begin{tabular}{|c|l|c|}
\hline Sl. No & \multicolumn{1}{|c|}{ Pig Breed } & Assigned Class \\
\hline 1 & Yorkshire & 1 \\
\hline 2 & Duroc & 2 \\
\hline 3 & Ghungroo & 3 \\
\hline 4 & Hampshire & 4 \\
\hline 5 & Mali & 5 \\
\hline
\end{tabular}

\begin{tabular}{|l|l|}
\hline Algorithm & Class assignment \\
\hline Input & Output of NNPB module \\
\hline Output & Class of pig Image \\
\hline Method & $\begin{array}{l}\text { Begin } \\
\text { If }\end{array}$ \\
Else If & $\begin{array}{l}\text { Output of NNPB module } \leq 1.5 \\
\text { Then Pig Image is in Class 1 } \\
\text { Output of NNPB module }>1.5 \& \& \text { Output } \\
\text { of NNPB module } \leq 2.5 \\
\text { Then Pig Image is in Class 2 }\end{array}$ \\
& $\begin{array}{l}\text { Else If } \\
\text { Output of NNPB module }>2.5 \& \& \text { Output } \\
\text { of NNPB module } \leq 3.5 \\
\text { Then Pig Image is in Class 3 } 3\end{array}$ \\
Else If & $\begin{array}{l}\text { Output of NNPB module }>3.5 \\
\text { \& Output of NNPB module } \leq 4.5 \\
\text { Then Pig Image is in Class 4 }\end{array}$ \\
Else & $\begin{array}{l}\text { Pig Image is in Class 5 } 5 \\
\text { End }\end{array}$ \\
\hline
\end{tabular}

The output of network produces numeric values. The output is categorized for classification of query pig images in classification module. The steps of classification are as follows.

\section{CAPTURING PIG IMAGES AND CREATING PIG BREED IMAGE DATABASE}

\section{Capturing of Individual Pig}

The images of pigs were captured in such a way that right or left side of their body was clearly visible is shown in Figure 5.

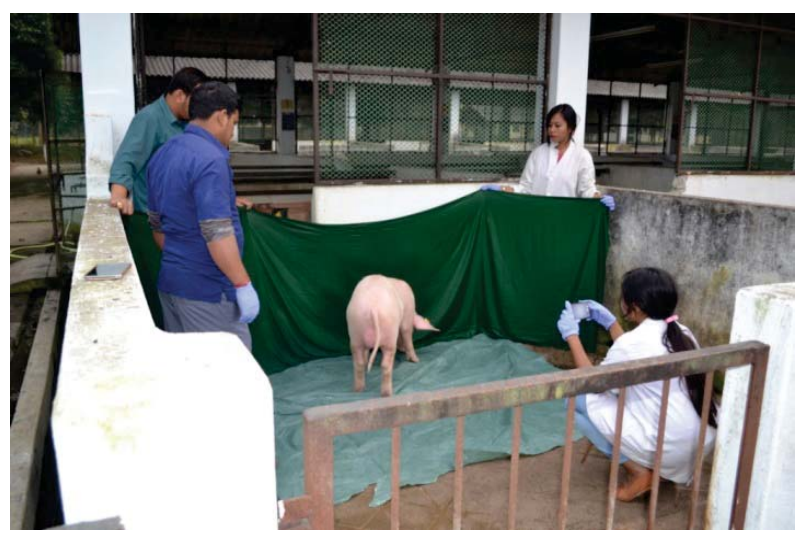

Fig. 5: Capturing the Pig Images

The steps were imposed for capturing the pigs as follows:

1. One green color curtain was placed over standing place and three sides of the pigs to have uniform background.

2. The camera was placed in one side parallel to pigs and keeping about 2 meters distance from it.

3. Lens of camera was hold in a position which was approximately in the middle of the length of the animal perpendicular to the median sagittal plane.

4. The lens was fixed few inches below the height of the animal to snapshot the entire visible parts of a pig.

5. The photos were captured using both smart phones' camera and DSLR camera under natural light in site profile fashion

\section{Pig Image Database}

Among the available breed of pig, five breeds, namely; Ghungroo, Mali, Hampshire, Duroc, and Yorkshire (Figure 6) were taken for study. To make use of breeds which are pure, 
images of individual animals from the said breeds were collected from organized farms, maintained by the premier research institutes of India, namely; 1) ICAR National Research Centre on pig, Rani, Assam, 2) ICAR Research Complex for NEH Region, Umiam, Meghalaya and 3) ICAR Research Complex for NEH Region, Tripura Centre, Tripura.
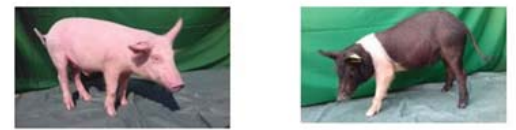

Yorkshire

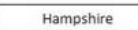

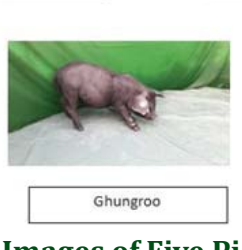
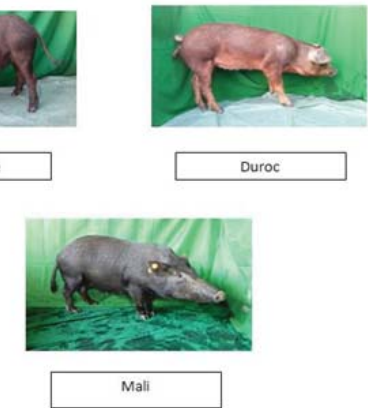

Fig. 6: Images of Five Pig Breeds with Uniform Background

\section{RESULT AND DISCUSSION}

The proposed pig breed classification model is composed of four sub modules as layered structure. The first module takes pig images as input and the classification module assigns class for input images. The result of each module is given separately as follows.

\section{SEgmentation OF CAPTURED IMAGES}

The 50 captured images from each of the five pig breeds named Yorkshire, Hampshire, Duroc, Ghungroo and Mali are used in this paper. All images are fed into the segmentation module and segmented image are produced. The segmented images are used in next layer as inputs. One sample segmented image from each pig breed is shown in Figure 7.

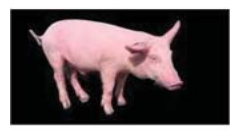

Yorkshire

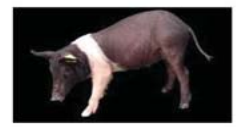

Hampshire

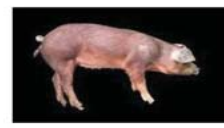

Duroc
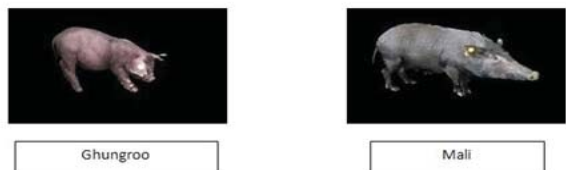

Fig. 7: Segmented Pig Images of Five Pig Breeds

In Figure 7, the background of all images is same and the site profiles are left or right. The background and profiles did not create any problem at the time of extracting statistical and color components from segmented images.

\section{EXTRACTED VALUES FROM STATISTICAL PARAMETERS AND COLOR COMPONENTS}

The segmented images from segmentation module are fed into Statistical components and color components retrieval module. The $\mathrm{H}, \mathrm{S}$ and $\mathrm{V}$ color component values are computed using RGB to HSV conversion algorithm. The Entropy, Standard deviation Mean, Median, Mode and Variance are computed using predefined equations. The five pig breeds are assigned into five classes and class are defined numerically as shown in Table 1 . The total 250 pigs from 5 pig breeds used in this experiment and ten parameters values from each segmented pig image are extracted. The outputs of this module are furnished as demo tabular form in Table 2. In table 2, extracted data from two segmented pig images from each breed are given and their corresponding breed and class values are furnished.

\section{BREED ClASSIFICATION ACCURACY USING NNPB MODULE}

The extracted data from statistical Parameters and Color Component Retrieval modules as furnished in Table 2 is fed into "Neural Network Pig Breed" neural network. The ten (10) parameter values named hue, saturation, value, entropy, standard deviation, mean, median, mode, sum and variance from each segmented image are used as input and the network produces its class value which is shown in Table 3.

The NNPB network is simulated using Matlab 2016. The NNPB module produced accuracy as $99.89 \%, 99.66 \%$ and $97.54 \%$ in training, validation and test respectively. The best performance, regression error plot, Error histogram and training state of NNPB are shown from Figure 8 to Figure 11. The performance of network is shown in Figure 8. It is observed that the MSE error is decreased due to decrease in weights during training by Levenberg-Marquardt backpropagation training algorithm. The blue, green and red lines are shown the MSE for the test, validation and training set, respectively. The best performance of validation is shown at epoch 10 where error is 0.093015 . The training of network is stopped at 16 epochs when the validation error reached a steady-state. The regression error plot is given at Figure 9. The data points are represented by circles and the lines represent the best fits between outputs and targets during training, validation and testing. The average $\mathrm{R}$ value for training, validation and testing is $0.98783(\geq 0.93)$ 


\section{Pig Breeds Classification using Neuro-Statistic Model}

represents that the fit is good for pig image classification. Error histogram is presented in Figure 10. The figure demonstrates the distribution of errors with the training, test and validation dataset. It is found that the maximum instances of MSE (around 93) are distributed close to zero line, which is shown by the orange line. Figure 11 depicts the training state of the network up to the moment of stopping. The gradient and $\mu$ values are 0.13285 and 0.00001 , respectively. It is shown that validation check at epoch 16 has few validation failures.

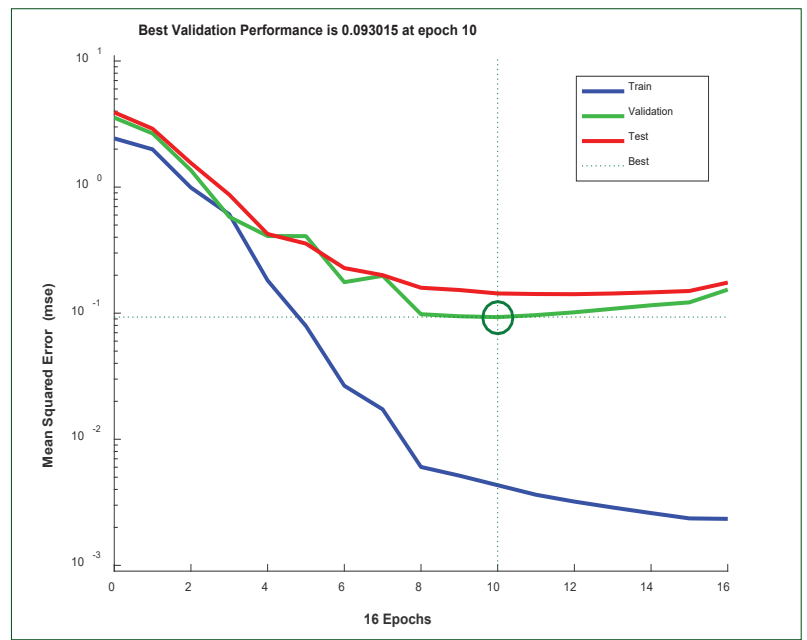

Fig. 8: Performance of Neural Network

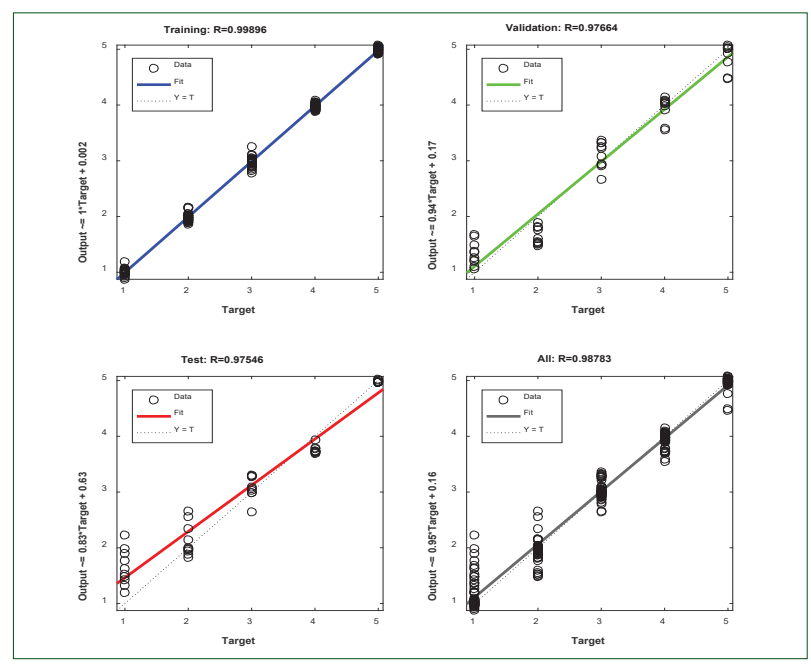

Fig. 9: Training, Test and validation for NNPB

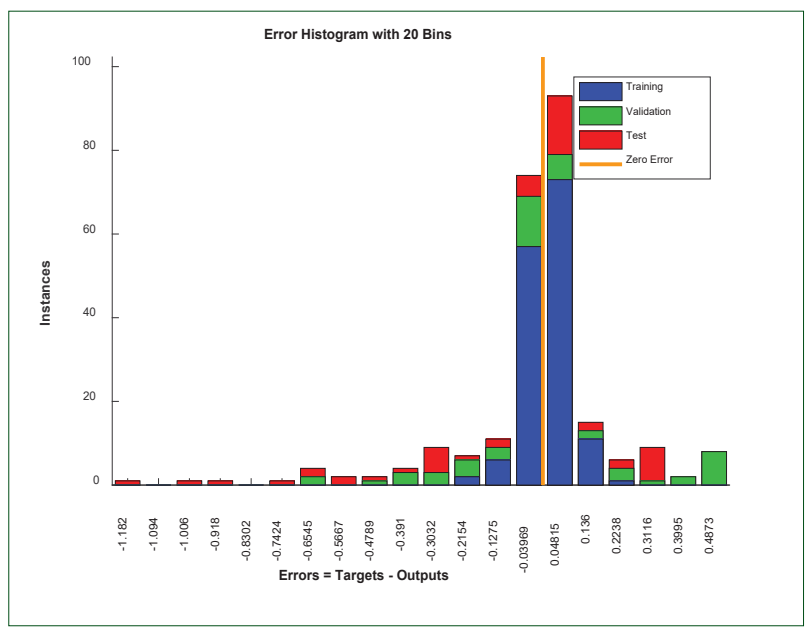

Fig. 10: Network Error Histogram

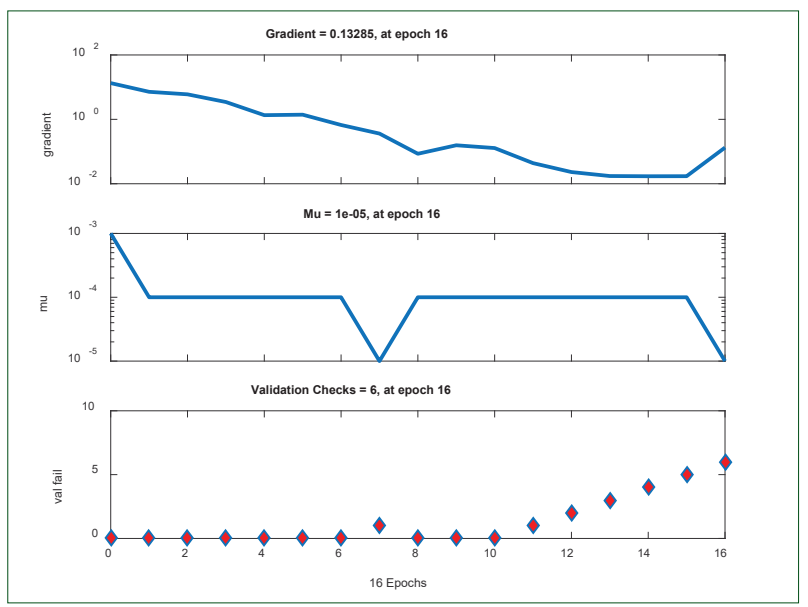

Fig. 11: Network Training State

\section{Classification Module And Breed PREDICTION}

The classification module is used for categorizing the output of NNPB network for classification based on algorithm given at section 4.5. 50 images are used to test the trained NNPB network. 10 images from each of the five breeds are used to test the network. The overall accuracy of proposed model is $90 \%$ is furnished in Table 4 and graphically represented in Figure 12. The breed wise accuracy graph is given in Figure 13. 
Table 2: Classification Result

\begin{tabular}{|c|l|c|c|c|}
\hline Sl. No & Pig Breed & $\begin{array}{c}\text { No. of Test } \\
\text { Images }\end{array}$ & $\begin{array}{c}\text { No. of } \\
\text { Classified } \\
\text { Successfully }\end{array}$ & $\begin{array}{c}\text { Accuracy } \\
\text { (\%) }\end{array}$ \\
\hline 01 & Yorkshire & 10 & 07 & 70 \\
\hline 02 & Duroc & 10 & 08 & 80 \\
\hline 03 & Ghungroo & 10 & 10 & 100 \\
\hline 04 & Hampshire & 10 & 10 & 100 \\
\hline 05 & Mali & 10 & 10 & 100 \\
\hline Overall & \multicolumn{1}{|l}{} & \\
\hline
\end{tabular}

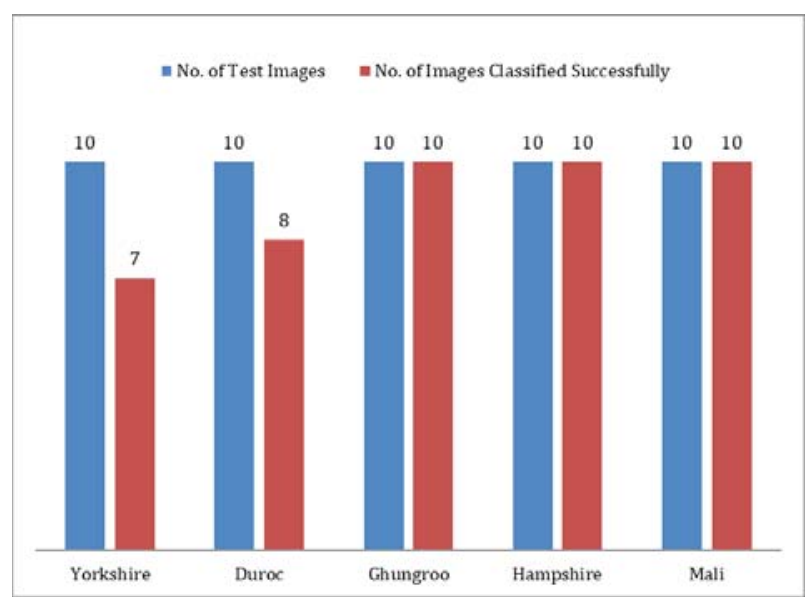

Fig. 12: No of Images Successfully Classified

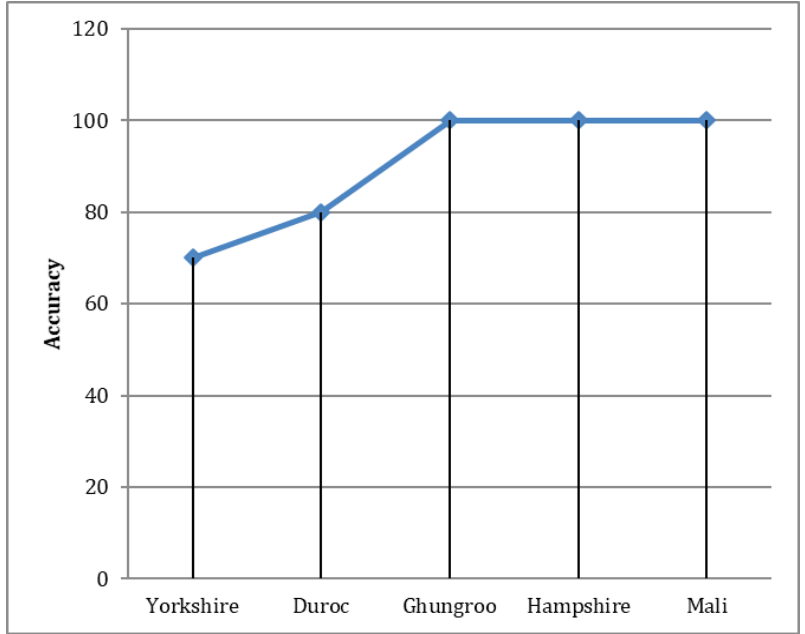

Fig. 13: Classification Accuracy

\section{CONCLUSION}

In this paper, Individual pig is classified based on the values of statistical parameters extracted from the content of its image. The individual pig from five pig breeds are captured at organized pig farms using mobile phones. The LevenbergMarquardt back propagation training algorithm with a minimum mean squared error and maximum correlation coefficient was found to be the best less than 10 epochs. The model is used to predict the breed of 50 pig images and the

Table 3: Statistical Parameter Values

\begin{tabular}{|c|c|c|c|c|c|c|c|c|c|c|c|c|c|}
\hline Sl.No & Image ID & $\mathbf{H}$ & $\mathbf{S}$ & V & Entropy & Std & Mean & Sum & Median & Mode & Variance & Breed & Class Value \\
\hline 1 & G1 & 0.152 & 0.107 & 0.074 & 2.374 & 24.748 & 16.342 & 1863.030 & 0.000 & 1.308 & 6519.171 & Ghungroo & 3 \\
\hline 2 & G2 & 0.145 & 0.105 & 0.074 & 2.290 & 24.628 & 16.313 & 1859.722 & 0.000 & 1.278 & 6635.538 & Ghungroo & 3 \\
\hline 3 & Y1 & 0.336 & 0.253 & 0.255 & 4.132 & 56.091 & 53.229 & 6068.157 & 0.096 & 31.222 & 9582.595 & Yorkshire & 1 \\
\hline 4 & Y2 & 0.278 & 0.236 & 0.203 & 3.516 & 55.982 & 41.956 & 4782.939 & 0.025 & 16.606 & 8683.902 & Yorkshire & 1 \\
\hline 5 & M1 & 0.142 & 0.062 & 0.141 & 3.599 & 45.877 & 35.085 & 3999.646 & 0.000 & 10.606 & 4572.518 & Mali & 5 \\
\hline 6 & M2 & 0.104 & 0.047 & 0.112 & 3.035 & 35.341 & 28.077 & 3200.798 & 0.000 & 5.409 & 4694.001 & Mali & 5 \\
\hline 7 & $\mathrm{H} 1$ & 0.224 & 0.230 & 0.159 & 4.112 & 36.803 & 33.704 & 3842.253 & 0.040 & 19.449 & 10009.343 & Hampshire & 4 \\
\hline 8 & $\mathrm{H} 2$ & 0.191 & 0.196 & 0.153 & 4.120 & 36.020 & 32.382 & 3691.535 & 0.010 & 18.854 & 9453.684 & Hampshire & 4 \\
\hline 9 & D1 & 0.073 & 0.259 & 0.259 & 4.092 & 56.073 & 52.393 & 5972.768 & 0.010 & 33.924 & 9889.457 & Duroc & 2 \\
\hline : & $:$ & : & : & & : & : & : & : & : & : & : & : & : \\
\hline : & $:$ & : & $:$ & : & : & $:$ & : & : & : & : & $:$ & $:$ & : \\
\hline 250 & D2 & 0.078 & 0.305 & 0.265 & 4.226 & 58.881 & 52.341 & 5966.879 & 0.040 & 27.641 & 8910.063 & Duroc & 2 \\
\hline
\end{tabular}




\section{Pig Breeds Classification using Neuro-Statistic Model}

Table 4: Input, Target and Actual Output of NNPB Network

\begin{tabular}{|c|c|c|c|c|c|c|c|c|c|c|c|c|c|}
\hline Sl.No & Image ID & $\mathbf{H}$ & $S$ & V & Entropy & Std & Mean & Sum & Median & Mode & Variance & $\begin{array}{l}\text { Target } \\
\text { output }\end{array}$ & $\begin{array}{l}\text { Actual } \\
\text { Output }\end{array}$ \\
\hline 1 & G1 & 0.152 & 0.107 & 0.074 & 2.374 & 24.748 & 16.342 & 1863.030 & 0.000 & 1.308 & 6519.171 & 3 & 3.0019 \\
\hline 2 & G2 & 0.145 & 0.105 & 0.074 & 2.290 & 24.628 & 16.313 & 1859.722 & 0.000 & 1.278 & 6635.538 & 3 & 2.9784 \\
\hline 3 & Y1 & 0.336 & 0.253 & 0.255 & 4.132 & 56.091 & 53.229 & 6068.157 & 0.096 & 31.222 & 9582.595 & 1 & 0.9932 \\
\hline 4 & Y2 & 0.278 & 0.236 & 0.203 & 3.516 & 55.982 & 41.956 & 4782.939 & 0.025 & 16.606 & 8683.902 & 1 & 0.9918 \\
\hline 5 & M1 & 0.142 & 0.062 & 0.141 & 3.599 & 45.877 & 35.085 & 3999.646 & 0.000 & 10.606 & 4572.518 & 5 & 4.9857 \\
\hline 6 & M2 & 0.104 & 0.047 & 0.112 & 3.035 & 35.341 & 28.077 & 3200.798 & 0.000 & 5.409 & 4694.001 & 5 & 5.0225 \\
\hline 7 & H1 & 0.224 & 0.230 & 0.159 & 4.112 & 36.803 & 33.704 & 3842.253 & 0.040 & 19.449 & 10009.343 & 4 & 4.0037 \\
\hline 8 & $\mathrm{H} 2$ & 0.191 & 0.196 & 0.153 & 4.120 & 36.020 & 32.382 & 3691.535 & 0.010 & 18.854 & 9453.684 & 4 & 3.9346 \\
\hline 9 & D1 & 0.073 & 0.259 & 0.259 & 4.092 & 56.073 & 52.393 & 5972.768 & 0.010 & 33.924 & 9889.457 & 2 & 1.9852 \\
\hline : & : & : & : & : & : & : & : & : & : & : & ; & : & : \\
\hline & : & : & : & : & : & : & : & : & : & : & : & : & \\
\hline 250 & D2 & 0.078 & 0.305 & 0.265 & 4.226 & 58.881 & 52.341 & 5966.879 & 0.040 & 27.641 & 8910.063 & 2 & 1.8660 \\
\hline
\end{tabular}

prediction accuracy is $90 \%$. Therefore, proposed model can be successfully used for the classification of pig breeds from individual pig images. The images from more pig breeds will be used in this experiment and more classification models will be used in future.

\section{ACKNOWLEDGEMENTS}

The authors would like to thank ITRA (Digital India Corporation, formerly Medialab Asia), MeitY, Govt. of India, for funding; Dr. Amitabha Bandyopadhyay, Senior Consultant, ITRA Ag \& Food for valuable suggestions; ICAR National Research Centre on pig, Rani, Assam, ICAR Research Complex for NEH Region, Umiam, Meghalaya and ICAR Research Complex for NEH Region, Tripura Centre, Tripura for permitting us to access their organized pig farms and Dr. Sourabh Kumar Das, Principal, Kalyani Government Engineering College for his continuous support.

\section{REFERENCES}

Akheela, A., \& Naz, H. (2015). Comparative analysis of Statistical Parameters of Finger Knuckle in Digital Image Processing. International Journal of Engineering and Computer Science.

Al-Ani, M. S., \& Alheeti, K. A. (2017). Precision statistical analysis of images based on brightness distribution. Advances in Science, Technology and Engineering Systems Journal, 99-104.

Garg, M., Malhotra, M., \& Singh, H. (2018). Statistical Feature Based Image Classification and Retrieval Using Trained Neural Classifiers. International Journal of Applied Engineering Research , 5766-5771.

Image Processing Fundamentals. (n.d.). Retrieved April 21, 202, from Vilnius University, Faculty of Mathematics and Informatics: http://www.mif.vu.lt/atpazinimas/dip/FIP/fip-Statisti. html\#Heading20

Islam, M. P., \& Morimoto, T. (2017). Non-linear autoregressive neural network approach for inside air temperature prediction of a pillar cooler. International journal of green energy , 141-149.

Jawale, A. (2019). Comparison of Image Classification Techniques: Binary and Multiclass using Convolutional Neural Network and Support Vector Machines. INFOCOMP Journal of Computer Science, 28-35. 
Kahou, S. E., \& Sulema, Y. (2010). Statistical approach to image classification. IEEE International Conference on Automation, Quality and Testing, Robotics (AQTR) (pp. 1-4). IEEE.

Kumar, V., \& Gupta, P. (2012). Importance of Statistical Measures in Digital Image Processing. International Journal of Emerging Technology and Advanced Engineering .

Pandey, N. K., Saxena, A., \& Bhatt, A. (2015). Performance Analysis of ANN models for the prediction of I.T. Professional's Occupational stress Using MATLAB. 4th International Conference on System Modeling \& Advancement in Research Trends (SMART). Moradabad.

Program to Change RGB color model to HSV color model. (n.d.). Retrieved April 21, 2020, from GeeksforGeeks: https://www. geeksforgeeks.org/program-change-rgb-color-model-hsvcolor-model/

Tahir, F., \& Fahiem, M. A. (2014). A statistical-textural-features based approach for classification of solid drugs using surface microscopic images. Computational and mathematical methods in medicine .
Thilagam, M., \& Arunesh, K. (2019). Content Based Image Retrieval using Statistical Parameters of a Medical Image. International Journal of Recent Technology and Engineering (IJRTE) .

Trnovszký, T., Kamencay, P., Orješek, R., Benčo, M., \& Sýkora, P. (2017). Animal recognition system based on convolutional neural network. DIGITAL IMAGE PROCESSING AND COMPUTER GRAPHICS

Variance \& Standard Deviation. (n.d.). Retrieved April 21, 2020, from Science Buddies: https://www.sciencebuddies.org/sciencefair-projects/science-fair/variance-and-standard-deviation

Wu, C. M., \& Chen, Y. C. (1992). Statistical feature matrix for texture analysis. CVGIP: Graphical Models and Image Processing , 407419.

Youssef, Y. B., Rabeh, A., Zbitou, J., \& Belaguid, A. (2014). Statistical features and classification of normal and abnormal mammograms. International Conference on Multimedia Computing and Systems (ICMCS) (pp. 448-452). IEEE. 